\title{
Predicting the limit of intramolecular H-Bonding with classical molecular dynamics
}

\section{Francesco Colizzi ${ }^{1 *}$, Adam Hospital ${ }^{1}$, Sanja Zivanovic ${ }^{1}$, and Modesto Orozco ${ }^{1,2 *}$}

\begin{abstract}
${ }^{1}$ Institute for Research in Biomedicine (IRB Barcelona), The Barcelona Institute of Science and Technology (BIST), Baldiri Reixac 10, Barcelona 08028, Spain 2 Departament de Bioquímica i Biomedicina. Facultat de Biologia. Universitat de Barcelona, Avgda Diagonal 647, Barcelona 08028, Spain
\end{abstract}

*Correspondence to: francesco.colizzi@irbbarcelona.org or modesto.orozco@irbbarcelona.org

\begin{abstract}
The energetics of intramolecular recognition processes are governed by the balance of pre-organization and flexibility that is often difficult to measure and hard to predict. Here, by using state-of-the-art classical molecular dynamics simulations, we predict and quantify the effective strength of intramolecular interactions between $\mathrm{H}$-bond donor and acceptor sites separated by a variable alkyl linker-in a variety of solvents and including crowded solutions. The fine balance of entropic and enthalpic contributions posits a solvent-dependent limit to the occurrence of intramolecular $\mathrm{H}$-bonding. Nevertheless, $\mathrm{H}$-bond free energies are rigidly shifted among different solvents with, for example, a systematic $\sim 13 \mathrm{~kJ} / \mathrm{mol}$ gap between water and chloroform. Molecular crowding shows little effects on thermodynamic equilibrium but it induces pronounced variations on $\mathrm{H}$-bond kinetics. The results are in quantitative agreement with available experimental measurements (in chloroform) and showcase a general strategy to interrogate molecular interactions in different environments, extending the limits of current experiments towards the prospective prediction of $\mathrm{H}$-bond interactions in pharmaceutical, agrochemical, and technological contexts.
\end{abstract}


Hydrogen bonds are ubiquitous interactions that drive molecular recognition, ${ }^{1,2}$ determine the properties of water and other polar solvents, ${ }^{3,4}$ play critical roles in enzyme catalysis ${ }^{5-7}$ and contribute to the structural stability ${ }^{8}$ as well as to the specificity of drug-target complexes. ${ }^{9,10}$ While $\mathrm{H}$-bonds are typically conceived as interactions between pairs of molecules, intramolecular hydrogen bonds are widespread in biological molecules, ${ }^{11,12}$ and are crucial in the design of new drugs and materials, ${ }^{13-17}$ including supramolecular machines. ${ }^{18-23}$ Unfortunately, characterization of intramolecular $\mathrm{H}$-bonds (and exploitation thereof) is still partial, most likely as a consequence of complexities that cloud the interpretation of experimental data obtained in large and flexible entities. ${ }^{11}$ Thus, it has long been known, for instance, that conformational flexibility posits a limit to the occurrence of intramolecular $\mathrm{H}$-bonds, ${ }^{24,25}$ but only recently Hubbard et al. ${ }^{26}$ have quantified such a limit using competition experiments in $\mathrm{CDCl}_{3}$ within a controlled molecular context. As a matter of fact, these measurements in deuterated chloroform ${ }^{26}$ set an unprecedented experimental reference in the field. Yet, in aqueous environments, the tiny population of $\mathrm{H}$ bonded species still challenges the limit of their experimental quantification and it remains unclear to which extent $\mathrm{H}$-bond measurements performed in noncompetitive solvents can be extended to polar solvents, such as water. ${ }^{27,28}$ As additional layer of complexity, the behavior of intramolecular $\mathrm{H}$-bonds in diluted water solutions could be altered by concentrated, or crowded, conditions that are likely to represent, better than diluted ones, the biological environment. ${ }^{29,30}$ In this context, computer simulations could bring about a major productivity leap providing quantitative information on interacting processes escaping from spectroscopic detection. Whereas ab initio simulations can provide unparalleled details on $\mathrm{H}$-bonds properties ${ }^{31,32}$, they require a great deal of computational power and their systematic application to flexible systems of varying complexity remains unpractical ${ }^{33}$. Finely tuned classical molecular dynamics (MD) simulations ${ }^{34}$, where electron and nuclear quantum ${ }^{35}$ effects are disregarded, offer a practical solution to efficiently sample the conformational space and have been successfully used to characterize $\mathrm{H}$-bond properties in a variety of contexts ranging from liquid water's kinetics ${ }^{3}$ to drug discovery. ${ }^{8}$ Yet, the capability of MD simulations to accurately quantify the balance between 
flexibility and self-assembly in intramolecular $\mathrm{H}$-bonding has not yet been investigated, as far as we are aware.

Here, building on recent experimental data ${ }^{26}$ we present a computational investigation on the influence of conformational flexibility on $\mathrm{H}$-bonding in a strictly intramolecular context using a series of model compounds ${ }^{26}$ (Figure 1A) immersed in a polar solvent (water), polar aprotic (tetrahydrofuran), and an apolar one (chloroform, for which partial experimental data ${ }^{26}$ is available for comparison and calibration). The effect of molecular crowding on $\mathrm{H}$-bonding behavior is also presented. The interactions between $\mathrm{H}$-bond donor and acceptor separated by a variable linker were sampled using classical MD simulations in the Hamiltonian Replica Exchange scheme ${ }^{36,37}$ (see Methods) and the energies compared to the number of rotatable bonds as well as to the solvent used. Results provide a quantitative solvent-transferable picture of the balance between hydrogen bonding and flexibility, and rise rules on the expected conformational landscape of molecules with potential to form intramolecular hydrogen bond and even on circularization propensity of long and flexible polymers. 
A)<smiles>Cn1c(=O)c2c(O)ccc(F)c2c2ccccc21</smiles>
1<smiles>CN(C)C(=O)c1cc(F)ccc1O</smiles>

2<smiles>CN(C)C(=O)Cc1cc(F)ccc1O</smiles>

3<smiles>CN(C)C(=O)CCc1cc(F)ccc1O</smiles>

4<smiles>CN(C)C(=O)CCCc1cc(F)ccc1O</smiles><smiles>CN(C)C(=O)CCCCCCCCc1cc(F)ccc1O</smiles>
10

B)

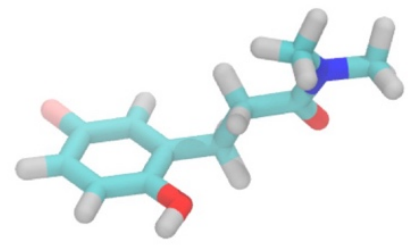

unfolded

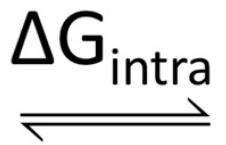

folded

Figure 1 Series of model compounds used to investigate the influence of a variable linker on intramolecular $\mathrm{H}$-bonding. Structure and nomenclature were inspired by Hubbard et al. ${ }^{26}$ A) Compound numbers 1-10 correspond to the number of rotatable bonds (in bold). B) Equilibrium in intramolecular folding.

Compounds 1-10 in Figure $1 \mathrm{~A}$ are expected to be in constant exchange between two major conformational ensembles in which the intramolecular $\mathrm{H}$ bond is either formed or broken (Figure 1B). Quantitative information of such conformational equilibrium can be obtained with MD simulations by sampling many transitions between folded and unfolded state and then reconstructing the free-energy profile along the intramolecular $\mathrm{H}$-bond distance, where a welldefined folded state can be discerned from the roughed unfolded ensemble (Figure 2). Notably, computer simulations are not limited by the nature of the solvent ${ }^{a}$ or by the tiny and short-lived population of the species of interest.

\footnotetext{
a Beyond the low solubility of 1-10 (predicted $\log S$ in water $<-1$ ), the experimental detection of conformational ratios in water seems unfeasible for these compounds even using an indirect competitive binding approach. Indeed, if the $\Delta \mathrm{G}$ of the competitive binding event $\left(\Delta \mathrm{G}_{\text {bind }}\right)$ is $>1 \mathrm{~kJ} / \mathrm{mol}$, no measurable binding is observed. ${ }^{26}$ Using a strong acceptor (e.g.: phosphine oxide), we estimate a $\Delta \mathrm{G}_{\text {bind }}$ of at least $+4 \mathrm{~kJ} / \mathrm{mol}$ even for compounds 7-10 that have a fairly accessible H-bond donor.
} 
Thus, the free energy associated to the intramolecular $\mathrm{H}$-bond in a given solvent, $\Delta G_{\text {intra }}$, can be obtained as $-k_{B} T \ln P($ folded $) / P$ (unfolded), where $k_{B}$ is the Boltzman constant, $T$ is the temperature and $P$ (folded) and $P$ (unfolded) is the population of folded and unfolded conformers, respectively.

Chloroform-derived results for compounds 1-10 show a non-trivial dependence between $\Delta G_{\text {intra }}$ and the donor-acceptor separation (Figure 3A, blue plot). Very encouragingly, such a complex dependence predicted by theoretical calculations quantitatively agrees with the experimental one derived from $\mathrm{NMR}^{26}$ (Figure 3A, gray plot), thus validating the parameters and the theoretical framework used herein. For very small linkers (2-3), strained intramolecular cycles are formed with an $\mathrm{H}$-bond angle around $100^{\circ}(\mathrm{C}=\mathrm{O} \cdots \mathrm{H}$ angle; shown Figure $3 \mathrm{~B}$ ) and a $\Delta G_{\text {intra }}$ of about $-5 \mathrm{~kJ} / \mathrm{mol}$. When 4 rotatable bonds separate donor and acceptors, the cyclization leads to a more favorable $\mathrm{H}$-bond with $\Delta G_{\text {intra }}$ of about $-8 \mathrm{~kJ} / \mathrm{mol}$ and the $\mathrm{C}=\mathrm{O} \cdots \mathrm{H}$ angle (Figure $3 \mathrm{~B}$ ) populating values around $120^{\circ}$, which is the most abundant acceptor directionality of $\mathrm{C}=\mathrm{O}$ observed in structural databases. ${ }^{38}$ Further addition of rotors gradually reduce the population of intramolecular $\mathrm{H}$-bonded species, leading to a pseudo plateau in $\Delta G_{\text {intra }}$ after 7 rotatable bonds. To further dissect the interaction, entropic and enthalpic contributions of the intramolecular $\mathrm{H}$-bond free energy were computed using Van't Hoff analysis (Figure 3C and Supporting Information, SI). We note that increments of the linker size progressively increase the entropy cost ($\mathrm{T} \Delta S_{\text {intra; }}$; Figure $3 \mathrm{C}$, blue triangles) of circularization, with an average penalty of $\sim 3 \mathrm{~kJ} / \mathrm{mol}$ per rotor. As the number of rotors goes beyond 6 , the entropic cost becomes larger than the enthalpic gain ( $\Delta H_{\text {intra, }}$, blue points in Figure $3 \mathrm{C}$ ) and the intramolecular $\mathrm{H}$-bond in chloroform is disfavored. Overall, for 7 or more rotors, the linker length enables the folded state to retain a conformational freedom that makes the resulting -T $\Delta S_{\text {intra }}$ values changing regime.

Contrary to chloroform solutions, the prevalence of circular topologies in water solvent is tiny as only a little population of intramolecular $\mathrm{H}$-bond is observed in the series of compounds studied here (Figure 3, red plots). As expected, water molecules competed avidly with the intramolecular $\mathrm{H}$-bond. As a result, the entropic cost of cyclization is higher than the enthalpic gain arising from intramolecular $\mathrm{H}$-bond formation (red plots in Figure $3 \mathrm{C}$ ). By comparing in-water 
and in-chloroform $\Delta G_{\text {intra }}$ values, we note that, depending on the environment, compounds 2-5 will populate different conformations and present different polarities-thus acting as molecular chameleons ${ }^{39,40}$ whose conformation can, for example, modulate membrane permeability. ${ }^{13,41, b}$ This behavior occurs in a size range relevant for small-molecule pharmaceuticals and should be considered for more accurate in silico prediction of ADME profiles. ${ }^{14-16}$

Very interestingly, the $\Delta G_{\text {intra }}$ vs linker-length profiles in water (red plots) and chloroform (blue plots) are clearly correlated (Figure 3A); the intramolecular interactions are systematically $12-15 \mathrm{~kJ} / \mathrm{mol}$ weaker in water than in chloroform, which is commensurate with the values proposed by other investigations. ${ }^{27}$ The thermodynamic quantities in Figure $3 \mathrm{C}$ show that the fine balance between enthalpy and entropy has a clear relationship with the solvent. However, the overall balance between enthalpy and entropy (i.e.: $\Delta G_{\text {intra}}$ ) controlling the formation of intramolecular cycles is, except for a rigid shifting factor, solvent independent. This is the case also when the $\Delta G_{\text {intra }}$ vs linker-length profile is collected in tetrahydrofuran (THF, Figure 3A, orange plot), suggesting that our conclusions can be extended to other pure solvents. We further note that such constant offset behavior on moving to different solvents, resembles the experimental data obtained when the competitive external binder was varied from the strong phosphine oxide acceptor to the weaker sulfinyl one. ${ }^{26}$

Finally, as intramolecular $\mathrm{H}$-bonding is of paramount importance in biology, we tested whether the above results and correlations hold also in highly concentrated, crowded conditions that can be reached, for instance, in the cellular environment (with up to $450 \mathrm{~g} / \mathrm{l}$ of macromolecules). ${ }^{29,30}$ Polyethyleneglycol (PEG) has been traditionally used as a reference crowding agent in the test tube ${ }^{42-44}$ and simulations. ${ }^{45,46}$ We thus measured $\Delta G_{\text {intra }}$ values for compounds 1-10 at increasing concentration of PEG in water (see Methods). Surprisingly, even at PEG concentrations as high as $450 \mathrm{~g} / \mathrm{l}$, the overall profile of $\Delta G_{\text {intra }}$ vs the number of rotatable bonds separating donor and acceptor remains unchanged with respect to dilute conditions (Figure S5). However, major differences appear in the potential of mean force associated to the formation of intramolecular $\mathrm{H}$-bonding, as crowding conditions increase the

\footnotetext{
${ }^{\mathrm{b}}$ Chloroform has a dielectric constant similar to that estimated to exist in the interior of a phospholipid bilayer.
} 
barrier to escape from, or arrive to, the folded state. As an example, H-bonded conformations of compound 4 are trapped by the increase of $3-4 \mathrm{~kJ} / \mathrm{mol}$ (green plot in Figure 4A) in the free energy barrier separating circularized and open states. This increment in the free energy barrier roughly translates (by Arrhenius relation) into a 4-times slower rate constant for the breaking of the intramolecular $\mathrm{H}$-bond with respect to infinitely diluted conditions (red plot in Figure 4A). On the other hand, also the folding process is slowed down as the metastable conformation observed at a distance of $\sim 0.4 \mathrm{~nm}$ can be stabilized by a bridging water molecule (Figure 4A) that becomes less available in crowded conditions. ${ }^{29}$ We argue that beyond the unspecific role of viscosity in decreasing molecular diffusion, ${ }^{29}$ the reduction of the number of water molecules able to diffuse into and away from the $\mathrm{H}$-bond in crowded conditions provides a mechanistic interpretation for the observed behavior (Figure 4B). This model agrees with, and provides a "single-interaction perspective" to, previous studies showing that alteration of water dynamics in crowded conditions slows down protein kinetics. ${ }^{47,48}$ The model also generalizes the behavior observed in ligand-receptor kinetics where the water accessibility of hydrogen bonding moieties can modulate ligand binding and unbinding rates. ${ }^{49-51}$

In summary, we have shown that the characterization, by carefully set up MD simulations, of intramolecular $\mathrm{H}$-bond energies in molecules of various flexibility finds quantitative agreement with NMR measurements and can be useful in the prediction of the conformational landscape of small molecules and polymers in conditions not accessible experimentally. The prevalence of intramolecular $\mathrm{H}$-bonding is modulated by a fine balance between intrinsic $\mathrm{H}$ bonding energy, the competition with solvent and the entropic cost of circularization. Interestingly, the overall balance between enthalpy and entropy is solvent independent and absolute values can be derived for solvent conditions different from the desired ones by applying a fix solvent-specific shifting factor, which facilitates extrapolation of our results. Molecular crowding can specifically slow down $\mathrm{H}$-bonding kinetics by shielding the interacting moieties from water molecules but does not, apparently, change the overall thermodynamics of folding, which again extends validity of our conclusions here. As to the transferability of the computational procedures used herein, we further remark that their application to other chemical systems, such as 
Schneider and coworkers' flexible dimers ${ }^{52}$, preliminary resulted in an impressive agreement with experimental measurements (see $\mathrm{SI}$ ). We are confident that our methodology and analysis provide a useful basis for a rational use of $\mathrm{H}$-bonding to guide conformations of flexible molecules in pharmaceutical, agrochemical, and technological contexts.

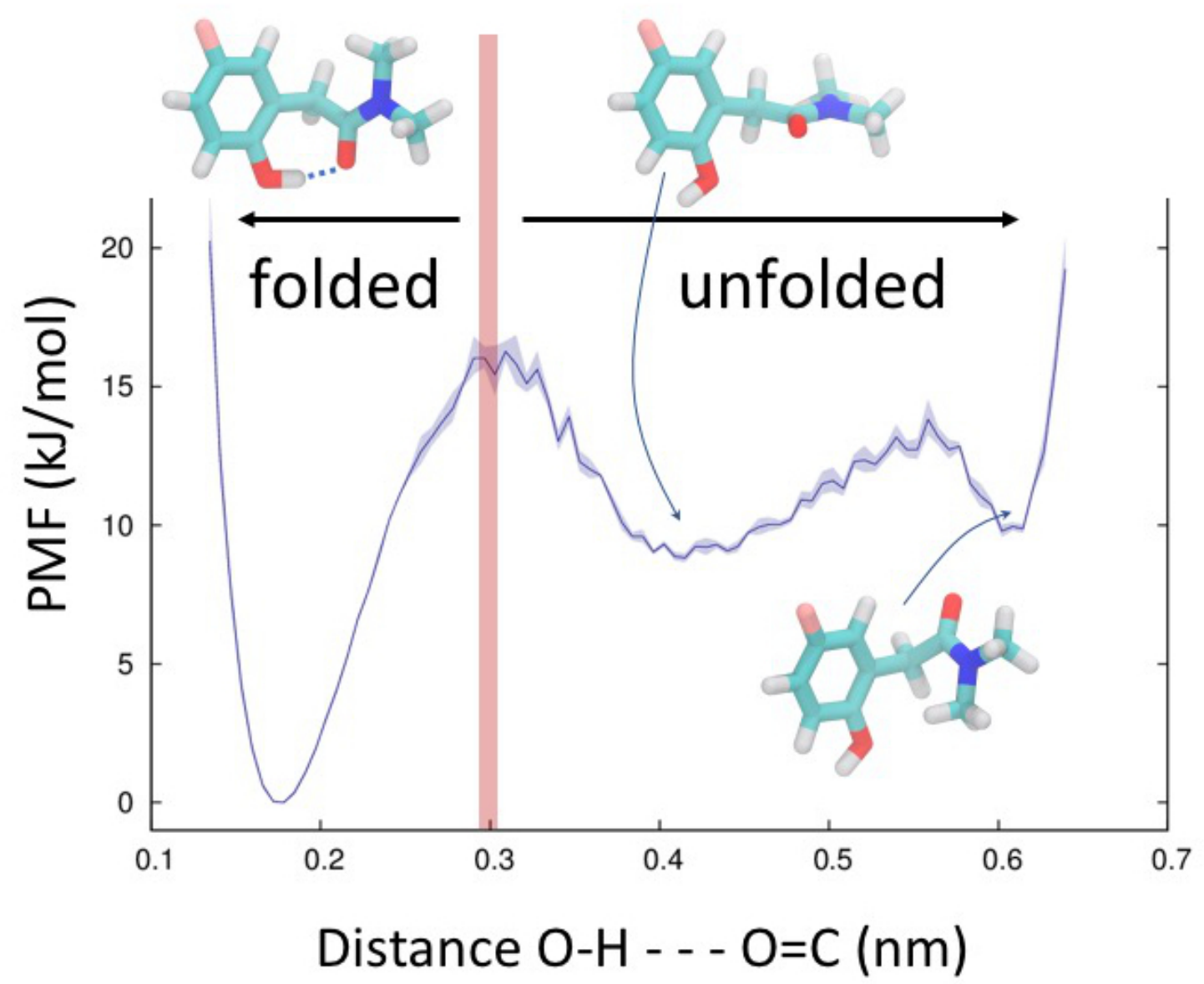

Figure 2 Explicative potential of mean force (PMF) of the folding process reconstructed as a function of the intramolecular distance between the hydroxylic hydrogen and the carbonylic oxygen. The energy barrier at $0.3 \mathrm{~nm}$ was used to discern folded from unfolded conformations for compounds 1-10. The shaded area represents the error estimated from block analysis. 

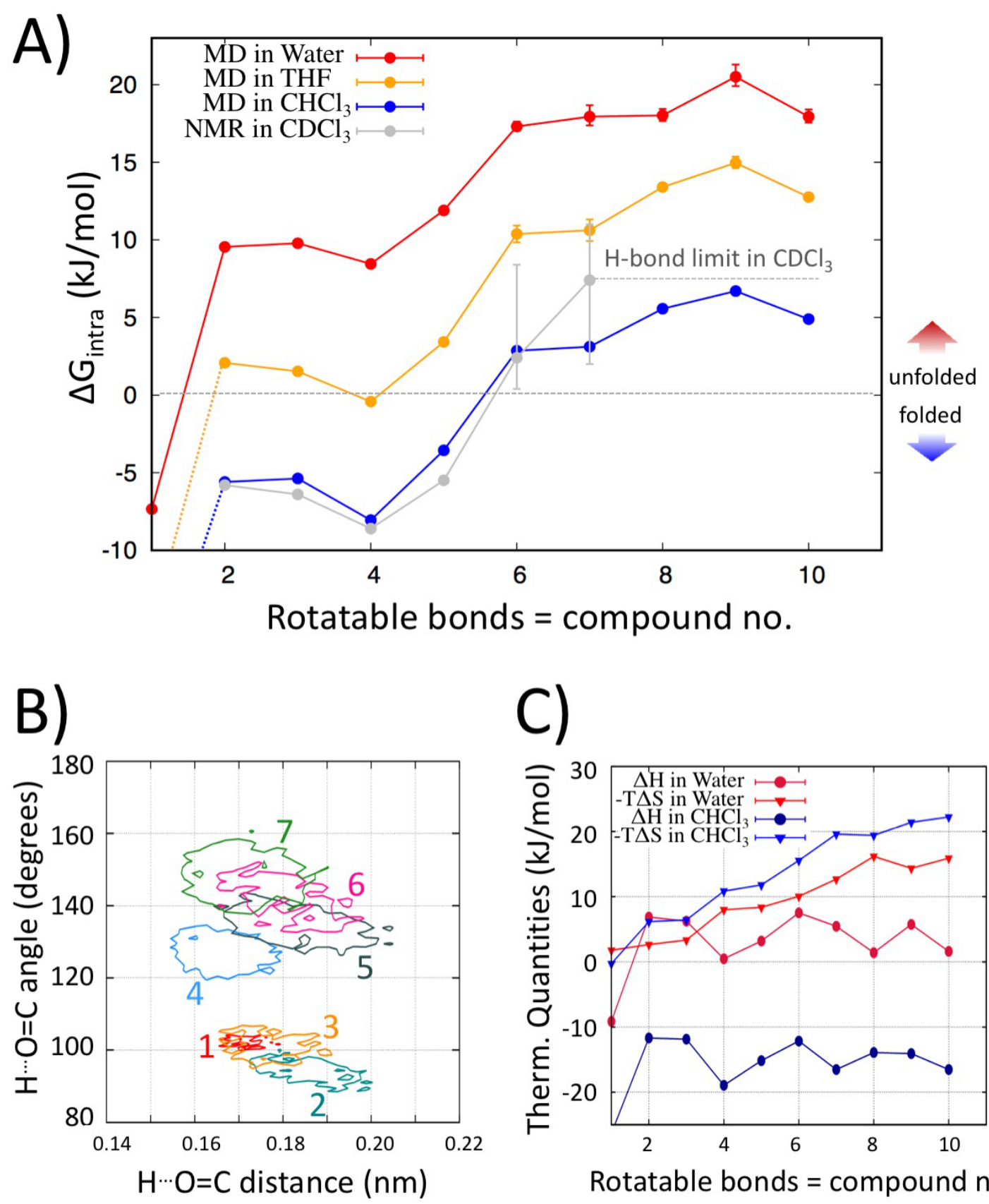

C)

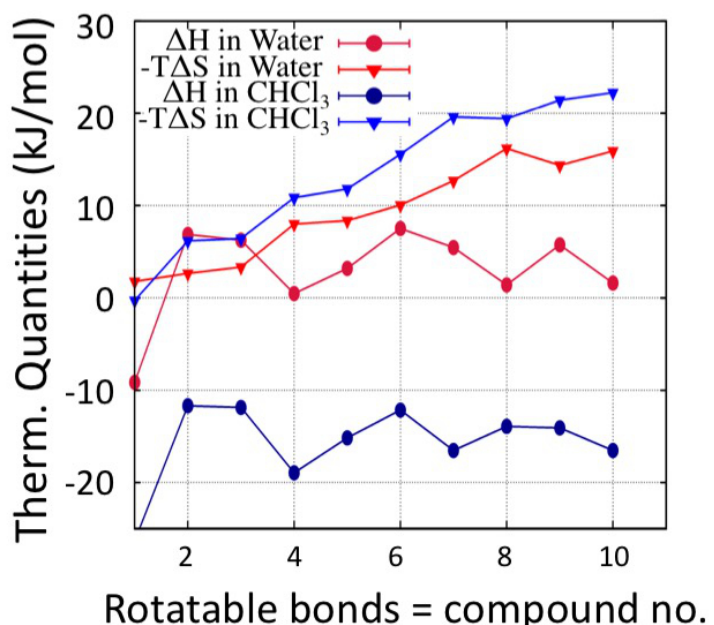

Figure 3 Thermodynamic characterization of intramolecular folding for compounds 1-10. A) Free energies of intramolecular folding $\left(\Delta G_{\text {intra }}\right)$ calculated in water (red line), tetrahydrofuran (THF, orange line), chloroform (blue line), and experimentally determined ${ }^{26}$ in deuterated chloroform (gray line). Error bars for simulation data estimated from block analysis. B) Geometrical features of the $\mathrm{H}$-bond formed in compounds 1-7. Contours are outlined for densities > 0.003 . For sake of clarity the entire analysis is reported in the Supporting Information (SI). C) We used a Van't Hoff analysis to separate enthalpic $\left(\Delta H_{\text {intra }}\right)$ and entropic (-T $\left.\Delta S_{\text {intra }}\right)$ contribution during the folding process (see SI). 


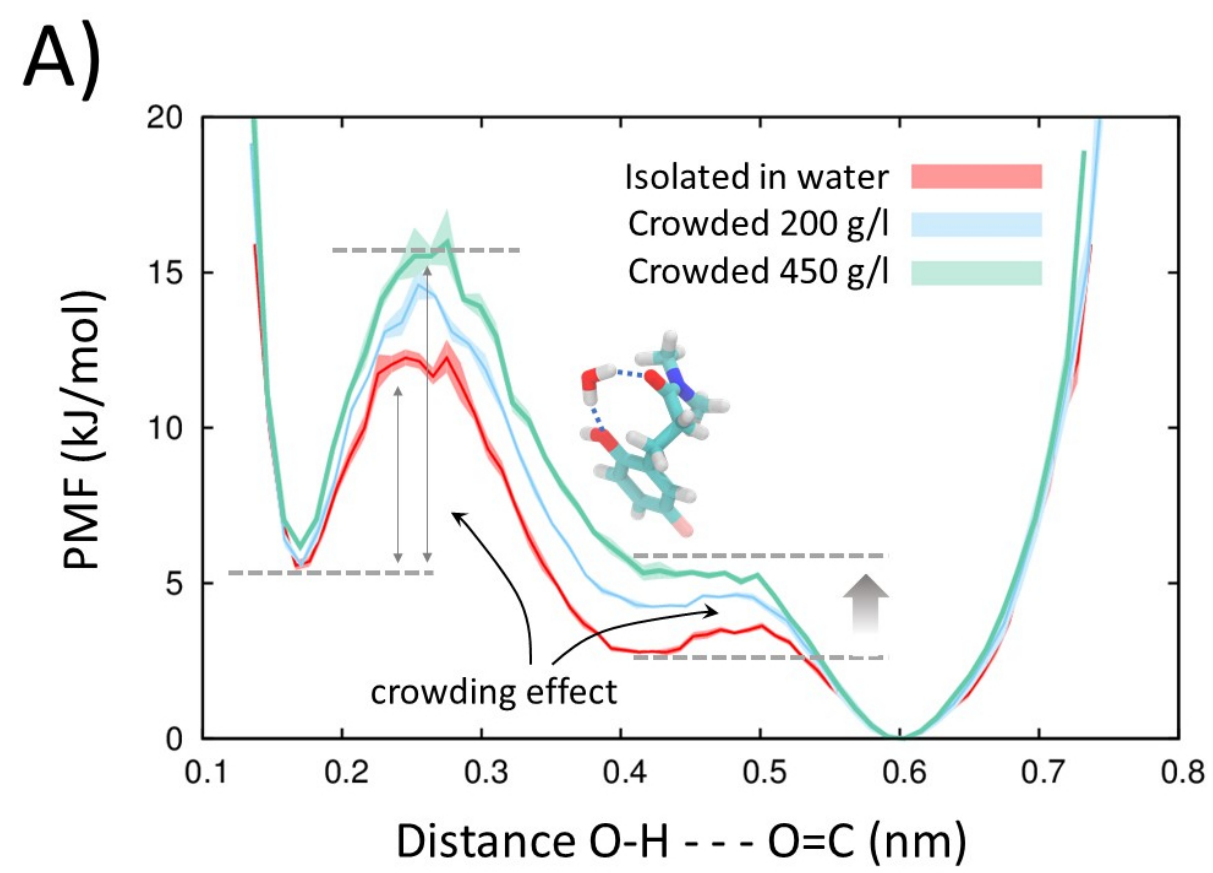

B)

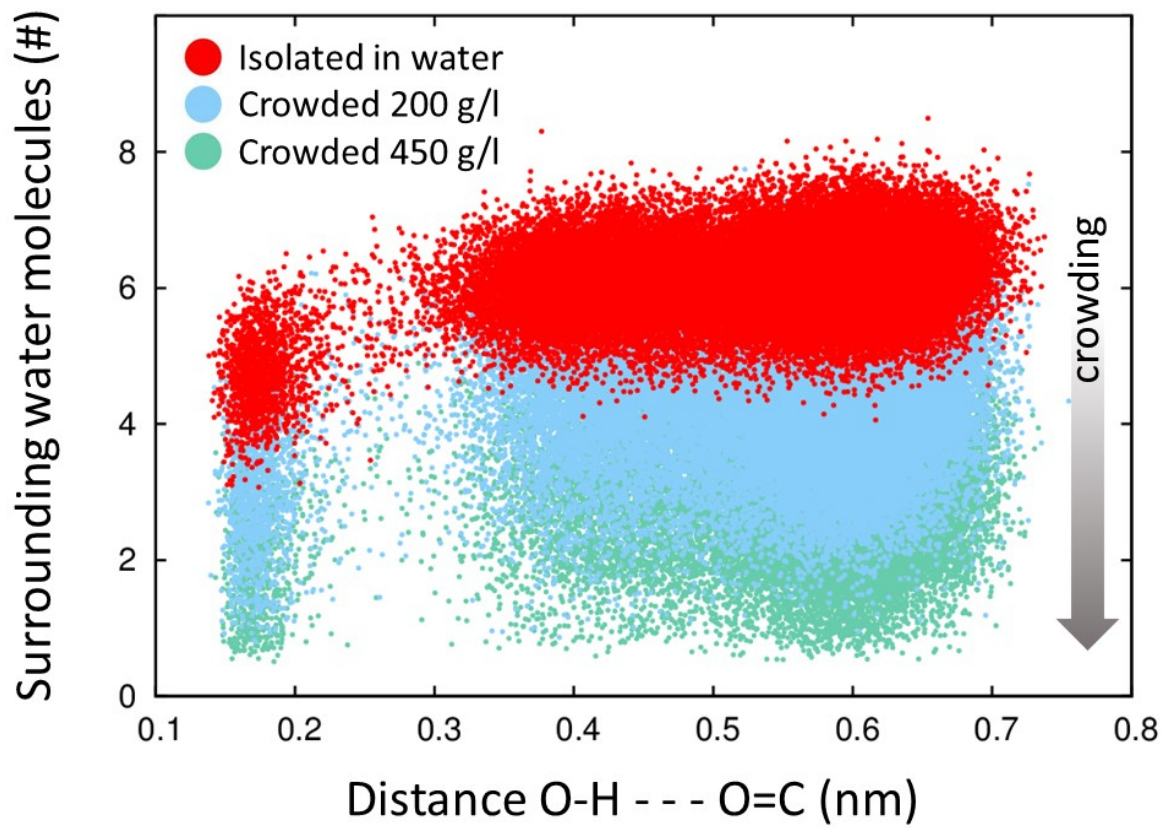

Figure 4 Effect of crowding on intramolecular $\mathrm{H}$-bonding. A) Variation of the potential of mean force (PMF) of the folding process of $\mathbf{4}$ depending on the concentration of the crowding agent (PEG). Crowded conditions (blue and green plots) show higher energy barriers with respect to infinitely diluted solutions (red plot). The metastable conformation at $\sim 0.4 \mathrm{~nm}$ can be stabilized by a bridging water molecule that becomes less available in crowded condition. The shaded area represents the error estimated from block analysis. B) Number of water molecules solvating the $\mathrm{H}$-bonding partners as a function of the intramolecular $\mathrm{H}$-bonding distance at different PEG concentration. Molecular crowding can specifically slow down $\mathrm{H}$-bonding kinetics by shielding the interacting moieties from water molecules. 


\section{Methods}

The 3-D structure of compounds 1-10 was built using Maestro from Schrödinger LCC. Compounds were modeled with the Generalized Amber Force Field $(\mathrm{GAFF})^{53}$ and partial atomic charges were assigned to the extended conformation using the Restrained Electrostatic Potential (RESP) fit ${ }^{54}$ at the HF/6-31G* level of theory using the RED server. ${ }^{55}$ See SI for further discussion on the charge model and the transferability of the computational approach. Parameters and topology files were prepared with Acpype. ${ }^{56}$ Compounds were solvated with a $0.8 \mathrm{~nm}$ thick box of TIP3P ${ }^{57}$ water molecules with periodic boundary conditions; for simulations in chloroform ${ }^{58,59}$ and tetrahydrofuran ${ }^{58,59}$ a $1.5 \mathrm{~nm}$-thick solvent box was used. Crowded environments were created by adding to the system box multiple copies of polyethylene glycol (PEG, $\mathrm{H}-\left(\mathrm{O}-\mathrm{CH}_{2}-\mathrm{CH}_{2}\right)_{n}-\mathrm{OH}$; with $\left.\mathrm{n}=2\right)$; PEG was modeled with GAFF and RESP charges.

Each system was equilibrated at constant pressure and temperature (1 atm, $298 \mathrm{~K}$ ); production runs were evolved at $298 \mathrm{~K}$ in the NVT ensemble with the velocity rescaling thermostat. ${ }^{60}$ Hamiltonian Replica EXchange (H-REX) simulations ${ }^{37}$ used 16 replicas with the scaling of all the atoms of the solute ${ }^{61}$ with values of $\lambda$ ranging from 1 to 0.59 . Exchanges were attempted every 500 steps. In infinitely diluted conditions (one solute molecule in the box), each replica run for 50 ns with a cumulative sampling time of 800 ns per compound per solvent used. In crowded conditions (with PEG) each replica either run for $100 \mathrm{~ns}$ (at $200 \mathrm{~g} / \mathrm{l}$ ) or for $200 \mathrm{~ns}$ (at $450 \mathrm{~g} / \mathrm{l}$ ). The effective temperature of each replica relates to the scaling factor $\lambda$ of the replica as $T_{\text {eff }}=298 \mathrm{~K} / \lambda$ and the values of InK $\mathrm{K}_{\text {intra }}$ were plotted against $1000 / \mathrm{T}_{\text {eff }}$ to estimate variations of entropy and enthalpy. All the simulations were run using GROMACS $4.6 .7^{62}$ patched with PLUMED $2.1^{63}$ and the H-REX implementation. ${ }^{36}$ Structural figures were made with $\mathrm{VMD}^{64}$ and chemical structures drawn with Marvin 18.1 (ChemAxon).

\section{Supporting Information}

Full methodological details on system set up, MD simulations and calculations. Supplementary data, including Figures S0-12. Supplementary discussion on the general validity and transferability of the computational approach used, the influence of the charge model on $\Delta G_{\text {intra. }}$, and the consistency of the results over a wide range of distance thresholds used to calculate $\Delta G_{\text {intra. }}$.

\section{Acknowledgment}

The authors thank Giovanni Bussi, Lorenzo Cupellini, Luis González, Montse Terrazas and all the members of the Molecular Modeling and Bioinformatics group for useful discussions and feedback. We thank José Antonio Alcántara 
for help with the use HPC infrastructures. F.C. has received funding from the European Union's Horizon 2020 research and innovation programme under the Marie Skłodowska-Curie grant agreement No 752415. This research has been supported by Spanish Ministry of Science (BFU2014-52864-R), the Catalan SGR (Grups Consolidats) and the BioExcel H2020 Project. The IRB Barcelona is recipient of a MINECO Severo Ochoa Award.

\section{References}

(1) Fersht, A. R. The Hydrogen Bond in Molecular Recognition. Trends Biochem. Sci. 1987, 12, 301-304.

(2) Desiraju, G. R. Chemistry beyond the Molecule. Nature 2001, 412 (6845), 397.

(3) Luzar, A.; Chandler, D. Hydrogen-Bond Kinetics in Liquid Water. Nature 1996, 379 (6560), 55.

(4) Wernet, P.; Nordlund, D.; Bergmann, U.; Cavalleri, M.; Odelius, M.; Ogasawara, H.; Näslund, L.-Å.; Hirsch, T. K.; Ojamäe, L.; Glatzel, P.; et al. The Structure of the First Coordination Shell in Liquid Water. Science (80-. ). 2004, 304 (5673), 995-999.

(5) Cleland, W. W.; Kreevoy, M. M. Low-Barrier Hydrogen Bonds and Enzymic Catalysis. Science (80-. ). 1994, 264 (5167), 1887-1890.

(6) Jeffrey, J. L.; Terrett, J. A.; MacMillan, D. W. C. O--H Hydrogen Bonding Promotes H-Atom Transfer from \$a $\$ \mathrm{C}-\mathrm{H}$ Bonds for C-Alkylation of Alcohols. Science (80-. ). 2015, 349 (6255), 1532-1536.

(7) Herschlag, D.; Pinney, M. Hydrogen Bonds: Simple after All? Biochemistry. 2018.

(8) Ruiz-Carmona, S.; Schmidtke, P.; Luque, F. J.; Baker, L.; Matassova, N.; Davis, B.; Roughley, S.; Murray, J.; Hubbard, R.; Barril, X. Dynamic Undocking and the Quasi-Bound State as Tools for Drug Discovery. Nat. Chem. 2017, 9 (3), 201.

(9) Bissantz, C.; Kuhn, B.; Stahl, M. A Medicinal Chemist s Guide to Molecular Interactions. J. Med. Chem. 2010, 53 (14), 5061-5084.

(10) Hunter, C. A. Quantifying Intermolecular Interactions: Guidelines for the Molecular Recognition Toolbox. Angew. Chemie Int. Ed. 2004, 43 (40), 5310-5324.

(11) Scheiner, S. Special Issue: Intramolecular Hydrogen Bonding 2017. Multidisciplinary Digital Publishing Institute 2017.

(12) McClellan, A. L. The Significance of Hydrogen Bonds in Biological Structures. J. Chem. Educ. 1967, 44 (9), 547.

(13) Gangwar, S.; Jois, S. D. S.; Siahaan, T. J.; Vander Velde, D. G.; Stella, V. J.; Borchardt, R. T. The Effect of Conformation on Membrane Permeability of an Acyloxyalkoxy-Linked Cyclic Prodrug of a Model Hexapeptide. Pharm. Res. 1996, 13 (11), 1657-1662.

(14) Kuhn, B.; Mohr, P.; Stahl, M. Intramolecular Hydrogen Bonding in Medicinal Chemistry. J. Med. Chem. 2010, 53 (6), 2601-2611.

(15) Alex, A.; Millan, D. S.; Perez, M.; Wakenhut, F.; Whitlock, G. A. 
Intramolecular Hydrogen Bonding to Improve Membrane Permeability and Absorption in beyond Rule of Five Chemical Space. Med. Chem.

Commun. 2011, 2 (7), 669-674.

(16) Caron, G.; Ermondi, G. Why We Need to Implement Intramolecular Hydrogen-Bonding Considerations in Drug Discovery. Future Science 2017.

(17) ten Brinke, G.; Ruokolainen, J.; Ikkala, O. Supramolecular Materials Based on Hydrogen-Bonded Polymers. In Hydrogen Bonded Polymers; Springer, 2007; pp 113-177.

(18) Panman, M. R.; Bakker, B. H.; Den Uyl, D.; Kay, E. R.; Leigh, D. A.; Buma, W. J.; Brouwer, A. M.; Geenevasen, J. A. J.; Woutersen, S. Water Lubricates Hydrogen-Bonded Molecular Machines. Nat. Chem. 2013, 5 (11), 929.

(19) Balzani, V.; Credi, A.; Raymo, F. M.; Stoddart, J. F. Artificial Molecular Machines. Angew. Chemie Int. Ed. 2000, 39 (19), 3348-3391.

(20) Erbas-Cakmak, S.; Leigh, D. A.; McTernan, C. T.; Nussbaumer, A. L. Artificial Molecular Machines. Chem. Rev 2015, 115 (18), 10081-10206.

(21) Yu, G.; Yung, B. C.; Zhou, Z.; Mao, Z.; Chen, X. Artificial Molecular Machines in Nanotheranostics. ACS Nano 2018, 12 (1), 7-12.

(22) Leigh, D. A.; Wong, J. K. Y.; Dehez, F.; Zerbetto, F. Unidirectional Rotation in a Mechanically Interlocked Molecular Rotor. Nature 2003, 424 (6945), 174.

(23) Dial, B. E.; Pellechia, P. J.; Smith, M. D.; Shimizu, K. D. Proton Grease: An Acid Accelerated Molecular Rotor. J. Am. Chem. Soc. 2012, 134 (8), 3675-3678.

(24) Galli, C.; Mandolini, L. The Role of Ring Strain on the Ease of Ring Closure of Bifunctional Chain Molecules. European J. Org. Chem. 2000, 2000 (18), 3117-3125.

(25) Illuminati, G.; Mandolini, L. Ring Closure Reactions of Bifunctional Chain Molecules. Acc. Chem. Res. 1981, 14 (4), 95-102.

(26) Hubbard, T. A.; Brown, A. J.; Bell, I. A. W.; Cockroft, S. L. The Limit of Intramolecular H-Bonding. J. Am. Chem. Soc. 2016, 138 (46), 1511415117.

(27) Cook, J. L.; Hunter, C. A.; Low, C. M. R.; Perez-Velasco, A.; Vinter, J. G. Solvent Effects on Hydrogen Bonding. Angew. Chemie Int. Ed. 2007, 46 (20), 3706-3709.

(28) Sun, H.; Hunter, C. A.; Navarro, C.; Turega, S. Relationship between Chemical Structure and Supramolecular Effective Molarity for Formation of Intramolecular H-Bonds. J. Am. Chem. Soc. 2013, 135 (35), 1312913141.

(29) Feig, M.; Yu, I.; Wang, P. H.; Nawrocki, G.; Sugita, Y. Crowding in Cellular Environments at an Atomistic Level from Computer Simulations. J. Phys. Chem. B 2017.

(30) Ellis, R. J.; Minton, A. P. Join the Crowd. Nature. 2003.

(31) Tuckerman, M. E.; Marx, D.; Klein, M. L.; Parrinello, M. On the Quantum Nature of the Shared Proton in Hydrogen Bonds. Science (80-. ). 1997.

(32) Li, X.-Z.; Walker, B.; Michaelides, A. Quantum Nature of the Hydrogen Bond. Proc. Natl. Acad. Sci. 2011.

(33) Amaro, R. E.; Mulholland, A. J. Bridging Biological and Chemical Complexity in the Search for Cures: Multiscale Methods in Drug Design. 
Nat Rev Chem 2018.

(34) Bottaro, S.; Lindorff-Larsen, K. Biophysical Experiments and Biomolecular Simulations: A Perfect Match? Science (80-. ). 2018.

(35) Markland, T. E.; Ceriotti, M. Nuclear Quantum Effects Enter the Mainstream. Nat. Rev. Chem. 2018.

(36) Bussi, G. Hamiltonian Replica Exchange in GROMACS: A Flexible Implementation. Mol. Phys. 2014, 112 (3-4), 379-384.

(37) Sugita, Y.; Okamoto, Y. Replica-Exchange Molecular Dynamics Method for Protein Folding. Chem. Phys. Lett. 1999, 314 (1-2), 141-151.

(38) Steiner, T. The Hydrogen Bond in the Solid State. Angew. Chemie Int. Ed. 2002, 41 (1), 48-76.

(39) Whitty, A.; Zhong, M.; Viarengo, L.; Beglov, D.; Hall, D. R.; Vajda, S. Quantifying the Chameleonic Properties of Macrocycles and Other HighMolecular-Weight Drugs. Drug Discov. Today 2016, 21 (5), 712-717.

(40) Carrupt, P. A.; Testa, B.; Bechalany, A.; El Tayar, N.; Descas, P.; Perrissoud, D. Morphine 6-Glucuronide and Morphine 3-Glucuronide as Molecular Chameleons with Unexpected Lipophilicity. J. Med. Chem. 1991, 34 (4), 1272-1275.

(41) Koehorst, R. B. M.; Spruijt, R. B.; Vergeldt, F. J.; Hemminga, M. A. Lipid Bilayer Topology of the Transmembrane \$a-Helix of M13 Major Coat Protein and Bilayer Polarity Profile by Site-Directed Fluorescence Spectroscopy. Biophys. J. 2004, 87 (3), 1445-1455.

(42) Kuznetsova, I. M.; Turoverov, K. K.; Uversky, V. N. What Macromolecular Crowding Can Do to a Protein. International Journal of Molecular Sciences. 2014.

(43) Kilburn, D.; Roh, J. H.; Guo, L.; Briber, R. M.; Woodson, S. A. Molecular Crowding Stabilizes Folded RNA Structure by the Excluded Volume Effect. J. Am. Chem. Soc. 2010.

(44) Phillip, Y.; Sherman, E.; Haran, G.; Schreiber, G. Common Crowding Agents Have Only a Small Effect on Protein-Protein Interactions. Biophys. J. 2009.

(45) Candotti, M.; Orozco, M. The Differential Response of Proteins to Macromolecular Crowding. PLoS Comput. Biol. 2016.

(46) Elcock, A. H. Models of Macromolecular Crowding Effects and the Need for Quantitative Comparisons with Experiment. Current Opinion in Structural Biology. 2010.

(47) Lu, C.; Prada-Gracia, D.; Rao, F. Structure and Dynamics of Water in Crowded Environments Slows down Peptide Conformational Changes. J. Chem. Phys. 2014.

(48) Harada, R.; Sugita, Y.; Feig, M. Protein Crowding Affects Hydration Structure and Dynamics. J. Am. Chem. Soc. 2012.

(49) Schmidtke, P.; Javier Luque, F.; Murray, J. B.; Barril, X. Shielded Hydrogen Bonds as Structural Determinants of Binding Kinetics: Application in Drug Design. J. Am. Chem. Soc. 2011.

(50) Pan, A. C.; Borhani, D. W.; Dror, R. O.; Shaw, D. E. Molecular Determinants of Drug-Receptor Binding Kinetics. Drug Discov Today 2013, 18 (13-14), 667-673.

(51) Bortolato, A.; Deflorian, F.; Weiss, D. R.; Mason, J. S. Decoding the Role of Water Dynamics in Ligand-Protein Unbinding: $C R F<\inf >1</ \operatorname{lnf}>R$ as a Test Case. J. Chem. Inf. Model. 2015. 
(52) Eblinger, F.; Schneider, H. J. Stabilities of Hydrogen-Bonded Supramolecular Complexes with Various Numbers of Single Bonds: Attempts to Quantify a Dogma in Host-Guest Chemistry. Angew. Chemie - Int. Ed. 1998.

(53) Wang, J.; Wolf, R. M.; Caldwell, J. W.; Kollman, P. A.; Case, D. A. Development and Testing of a General Amber Force Field. J. Comput. Chem. 2004, 25 (9), 1157-1174.

(54) Bayly, C. I.; Cieplak, P.; Cornell, W.; Kollman, P. A. A Well-Behaved Electrostatic Potential Based Method Using Charge Restraints for Deriving Atomic Charges: The \{RESP $\}$ Model. J. Phys. Chem. 1993, 97 (40), 10269-10280.

(55) Vanquelef, E.; Simon, S.; Marquant, G.; Garcia, E.; Klimerak, G.; Delepine, J. C.; Cieplak, P.; Dupradeau, F.-Y. R.E.D. Server: A Web Service for Deriving RESP and ESP Charges and Building Force Field Libraries for New Molecules and Molecular Fragments. Nucleic Acids Res. 2011, 39 (Web Server issue), W511--W517.

(56) da Silva, A. W.; Vranken, W. F. ACPYPE - AnteChamber PYthon Parser InterfacE. BMC Res. Notes 2012, 5, 367.

(57) Jorgensen, W. L. Quantum and Statistical Mechanical Studies of Liquids. 10. Transferable Intermolecular Potential Functions for Water, Alcohols, and Ethers. Application to Liquid Water. J. Am. Chem. Soc. 1981, 103 (2), 335-340.

(58) Caleman, C.; van Maaren, P. J.; Hong, M.; Hub, J. S.; Costa, L. T.; van der Spoel, D. Force Field Benchmark of Organic Liquids: Density, Enthalpy of Vaporization, Heat Capacities, Surface Tension, Isothermal Compressibility, Volumetric Expansion Coefficient, and Dielectric Constant. J. Chem. Theory Comput. 2012, 8 (1), 61-74.

(59) Zhang, J.; Tuguldur, B.; Van Der Spoel, D. Force Field Benchmark of Organic Liquids. 2. Gibbs Energy of Solvation. J. Chem. Inf. Model. 2015.

(60) Bussi, G.; Donadio, D.; Parrinello, M. Canonical Sampling through Velocity Rescaling. J. Chem. Phys. 2007, 126 (1), 14101.

(61) Wang, L.; Friesner, R. A.; Berne, B. J. Replica Exchange with Solute Scaling: A More Efficient Version of Replica Exchange with Solute Tempering (REST2). J. Phys. Chem. B 2011, 115 (30), 9431-9438.

(62) Hess, B.; Kutzner, C.; Van Der Spoel, D.; Lindahl, E. GROMACS 4: Algorithms for Highly Efficient, Load-Balanced, and Scalable Molecular Simulation. J. Chem. Theory Comput. 2008, 4 (3), 435-447.

(63) Tribello, G. A.; Bonomi, M.; Branduardi, D.; Camilloni, C.; Bussi, G. PLUMED 2: New Feathers for an Old Bird. Comput. Phys. Commun. 2014, 185 (2), 604-613.

(64) Humphrey, W.; Dalke, A.; Schulten, K. VMD: Visual Molecular Dynamics. J. Mol. Graph. 1996, 14 (1), 27-28,33-38. 
Graphical Abstract
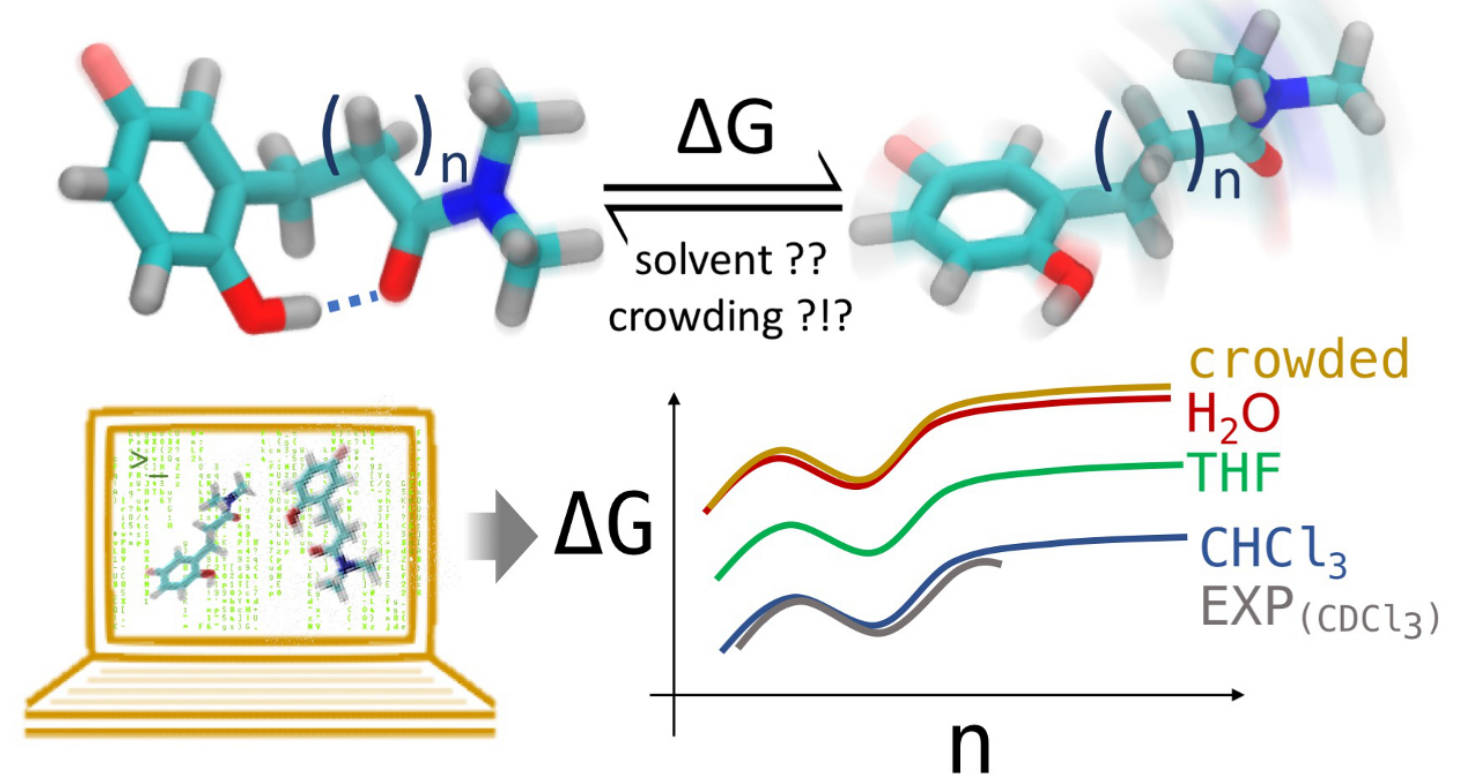